\title{
POWER CONSUMPTION FOR MIXING OF SHEAR-THINNING FLUIDS
}

\author{
ADINA GHIRIŞAN (MICLĂUŞ) ${ }^{\mathrm{a}}$ and VASILE MICLĂUŞ ${ }^{\mathrm{a}^{*}}$
}

\begin{abstract}
The mixing/agitation of time independent non-Newtonian fluids can be a challenge in many technological processes. In the present study, the influence of rheological parameters, $\mathrm{K}$ and $\mathrm{n}$, on Reynolds number, power number and power consumption, for different concentration of carboxymethyl cellulose (CMC) solutions, 1\%, 2\%, 3\%, $4 \%$ and 5\%, with shear-thinning behaviour, was analysed. The power consumption obtained by theoretical model and by an appropriate curve $P o=f(R e)$ from experimental data, found in literature, was compared. The results have shown good agreement between calculated power number and the graphically determined values, and also minimal power consumption values comparable with those founded for the agitation of non-Newtonian liquids.
\end{abstract}

Keywords: mixing, agitation, Reynolds number, power number, power consumption, non-Newtonian fluids, shear-thinning fluids.

\section{INTRODUCTION}

Agitation/mixing is one of the most common operations in the chemical, biochemical, polymer processing, and other industries. Almost all manufacturing processes entail some sort of mixing, and the operation may constitute a considerable proportion of the total processing time. Mixing is a physical process which aims to reduce the gradients of concentration, temperature and other properties in fluids.

In mixing, there are two types of problems to be considered - how to design and select mixing equipment for a given duty and how to assess whether an available mixer is suitable for a particular application. In both cases, the following aspects of the mixing process must be evaluated: the

\footnotetext{
a Babeş-Bolyai University, Faculty of Chemistry and Chemical Engineering, 11 Arany Janos str., RO-400028, Cluj-Napoca, Romania,

*Corresponding author: vasile.miclaus@ubbcluj.ro
} 
mechanisms of mixing, mixing time, scale-up or similarity criteria, specific power consumption, shear rate distribution and flow characteristics [1, 2].

From a practical point of view, power consumption is one of the most important parameter in the design of stirred vessels.

Mixing power for non-aerated Newtonian fluids depends on stirrer speed, $N$, the impeller diameter and geometry, D, and fluid properties such as density $\rho$, and viscosity $\eta$, Equation 1 [3].

$$
\begin{aligned}
& P o=\frac{P}{\rho \cdot N^{3} \cdot D^{5}} \\
& P=P o \cdot \rho \cdot N^{3} \cdot D^{5}
\end{aligned}
$$

where: $\mathrm{Po}$ is the power number (-), and $\mathrm{P}$ - power consumption (W).

The power number $\mathrm{Po}$, a dimensionless group, has a significant effect on the definition of power consumption.

The hydrodynamics is strongly dependent on the nature of the fluids involved in the mixing system. Shear-thinning fluids are a class of nonNewtonian fluids, as in the present study, carboxymethyl cellulose (CMC) solution with different concentrations [4].

Carboxymethyl cellulose (CMC) is a water-soluble cellulose derivative with many applications in food, pharmaceutical, cosmetics, detergent, textile, paper and many other industries. It is a white to off-white, non-toxic, odourless and biodegradable powder. CMC is used to enhance the viscosity, to control the rheology of a solution, to avoid separation of water from a suspension, and to improve the surface barrier properties [5].

The rheological properties of CMC depend on the concentration of polymer and the degree of substitution, which varies from 0.5-1.2 [6].

The prediction of power consumption for agitation of time-independent non-Newtonian fluid, at a desired impeller speed, may be evaluated considering the dependence of power number on Reynolds and Froude number, $P o=f(R e, F r)$.

The value of the Reynolds number is given by:

$$
\mathrm{Re}=\frac{\rho \cdot \mathrm{N} \cdot \mathrm{D}^{2}}{\eta_{\text {eff }}}
$$

The corresponding apparent viscosity $\eta_{\text {eff }}$ can be evaluated, either from a flow curve, or by means of the appropriate viscosity model (e.g. power law model). 
Depending on the value of Reynolds number it was considered the laminar regime for $\operatorname{Re}<10$, when $\mathrm{Po} \cong 1 / \mathrm{Re}$, turbulent regime $\operatorname{Re}>10^{3}-10^{4}$, when Po becomes independent of Reynolds number, and between them, the transition regime, when both density and the viscosity affect power requirements [1]. The influence of Froude number was neglected in the present estimation; do to the fact that it is generally important only when vortex formation occurs and Reynolds number is higher than 300 .

It is known, that as the liquid viscosity or constancy increases, the tendency for vortex formation decreases.

The aim of the present work is to evaluate the fluid rheological parameters on Reynolds criteria, power number Po and specific power consumption $\mathrm{P}$, using different types of stirrers for mixing of $\mathrm{CMC}$ solutions with shear-thinning behaviour.

\section{RESULTS AND DISCUSSION}

In the present study, the Reynolds number was calculate with the corresponding effective viscosity, $\eta_{\text {eff, }}$ evaluated by means of the power viscosity model:

$$
\eta_{\text {eff }}=\mathrm{K} \cdot \dot{\gamma}_{\text {avg }}^{\mathrm{n}-1}
$$

The mean shear rate was for the first time estimated by the Metzner and Otto [7] and correlated with rotational speed by Equation (4):

$$
\dot{\gamma}_{\text {avg }}=\mathrm{k}_{\mathrm{s}} \cdot \mathrm{N}
$$

where: $k_{s}$ is the shearing constant, normally dependent on the type of the stirrer and vessel configuration, and $\mathrm{N}$ is the rotation speed of stirrer (rot/s).

The Metzner-Otto method is a widely used method to design impellers for non-Newtonian fluids applications.

For the agitation of shear-thinning liquids by turbines, propellers and paddles, Skelland [8] suggest that the shearing constant $\mathrm{k}_{\mathrm{s}}$ lies approximately in the range $10-13$.

Based on the Godfrey [9] and Wang [10] observations, there are no scale-problems, $k_{s}$ is independent on the equipment size, and fluid properties.

In the present study, three types of stirrers, paddle, propeller and turbine, are analysed. For each type, the value of $k_{s}$ is chosen in agreement with the literature data, 10.0 for 2-badles paddle, 10.0 for propeller and 11.5 for turbine $[1,11]$. 
Table 1 contains the obtained values of rheological parameters, $\mathrm{K}$ and $\mathrm{n}$, for CMC solutions with 1, 2, 3, 4 and 5 , weight $\%$, based on measured data.

The obtained values of flow index, $\mathrm{n}$ smaller than 1 , indicate the shear-thinning or pseudo-plastic behaviour of all CMC solutions. The values are closer to those founded in literature [12]. As it was expected, with the increase of $\mathrm{CMC}$ concentration the index flow $\mathrm{n}$ decreases and the material constancy $\mathrm{K}$ increases.

Table 1. Rheological parameters of CMC solutions

\begin{tabular}{|c|c|c|}
\hline CMC (\%) & $K\left(P a \cdot s^{n}\right)$ & n (-) \\
\hline 1.00 & 0.110 & 0.9000 \\
\hline 2.00 & 0.910 & 0.7400 \\
\hline 3.00 & 3.035 & 0.6300 \\
\hline 4.00 & 9.250 & 0.5626 \\
\hline 5.00 & 28.000 & 0.5200 \\
\hline
\end{tabular}

The influence of the rheological parameters $\mathrm{K}$ and $\mathrm{n}$ on the Reynolds number, calculated with the Equation (2), is shown in Figure 1.

For all types of stirrers evaluated in this article, paddle, marine propeller and turbine (Figure 1), the decrease of the Reynolds number with the increase of material constancy $\mathrm{K}$ and the decrease of flow index $\mathrm{n}$, is correlated with $\mathrm{CMC}$ concentration.

The agitation of non-Newtonian fluids is recommended to be done at low Reynolds, in laminar or creeping-flow region. In the present study, the Reynolds number was calculated as for laminar model up to $\operatorname{Re}<50$, obtained for CMC concentration higher than $2 \%$.

The prediction of power consumption for agitation of our solutions is evaluated by the Equation (5):

$$
\mathrm{P}=\mathrm{k}_{1} \cdot \eta_{\text {eff }} \mathrm{N}^{2} \cdot \mathrm{D}^{3}=\mathrm{k}_{1} \cdot \mathrm{K} \cdot\left(\mathrm{k}_{\mathrm{s}} \cdot \mathrm{N}\right)^{\mathrm{n}-1} \cdot \mathrm{N}^{2} \cdot \mathrm{D}^{3}
$$

The values of power number determined directly as a function of Reynolds number, $\mathrm{Po}=\mathrm{k}_{1} / \mathrm{Re}$, and the power consumption $\mathrm{P}$ mathematically calculated by Equation 5, are compared with those obtained by graphically method from literature [1] in Table 2. 


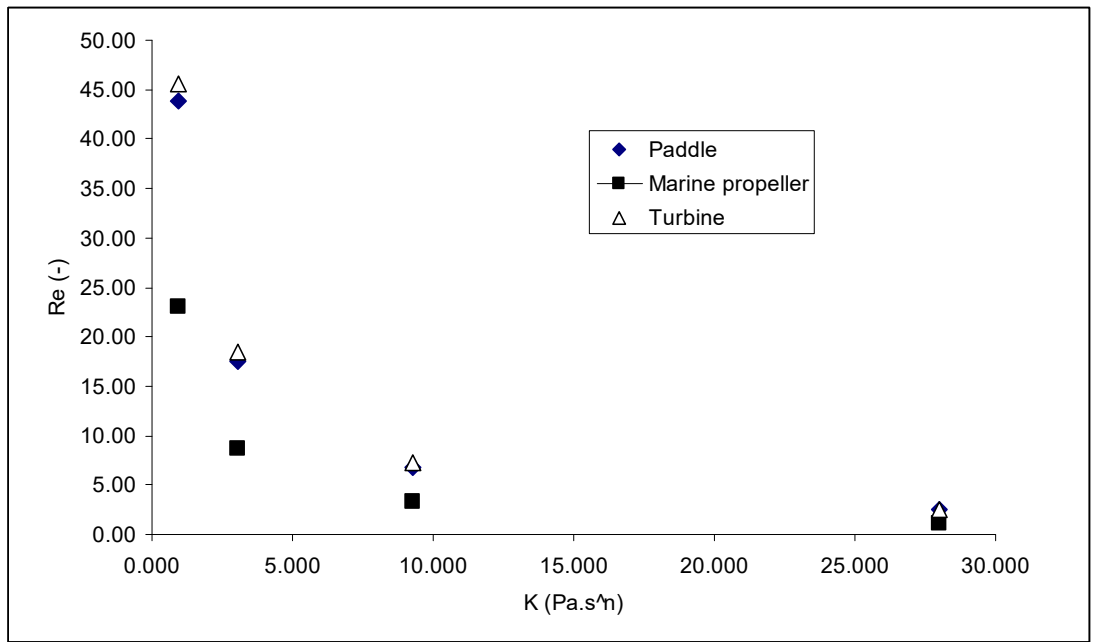

a.

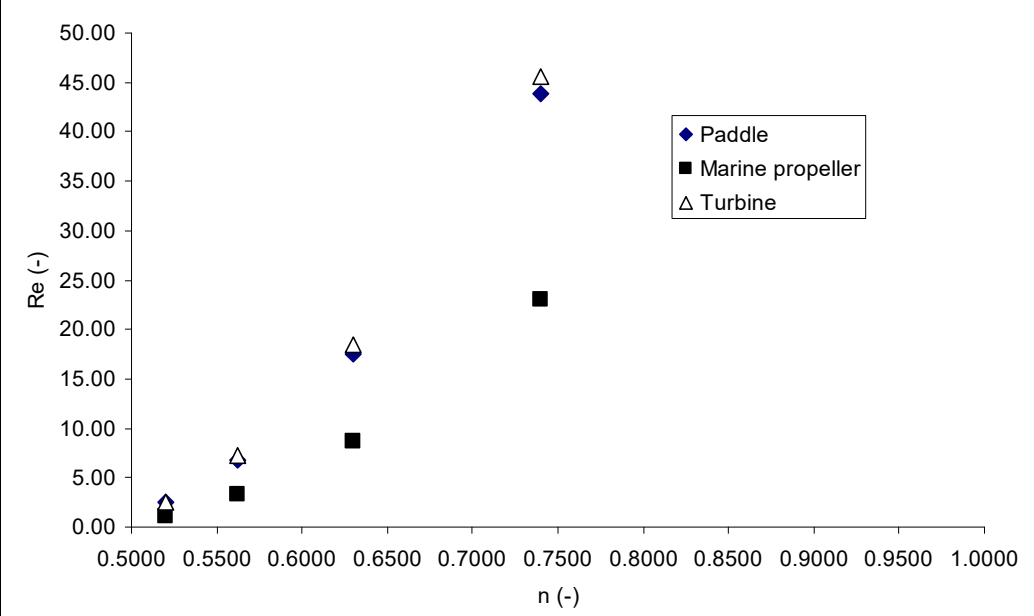

b.

Figure 1. The influence of the material constancy $K(a)$ and flow index $n$ (b) on Reynolds number

The constant $k_{1}$ dependent only on the impeller type has the values presented in Table 2 [8]. 
Table 2. Values of power number and power consumption

\begin{tabular}{|c|c|c|c|c|c|c|c|c|}
\hline $\begin{array}{l}\text { CMC } \\
(\%)\end{array}$ & $\begin{array}{c}\mathrm{N} \\
\text { (rot/s) }\end{array}$ & $\begin{array}{c}D \\
\text { (m) }\end{array}$ & $\begin{array}{l}\mathbf{k}_{1} \\
(-)\end{array}$ & $\begin{array}{l}\operatorname{Re} \\
(-)\end{array}$ & $\begin{array}{c}\text { Po } \\
\text { math } \\
(-)\end{array}$ & $\begin{array}{c}P \\
\text { math } \\
\text { (W) }\end{array}$ & $\begin{array}{c}\text { Po } \\
\text { graph } \\
(-)\end{array}$ & $\begin{array}{c}P \\
\text { graph } \\
\text { (W) }\end{array}$ \\
\hline \multicolumn{9}{|c|}{ 2-blades Paddle } \\
\hline 2 & 1.00 & 0.15 & 35 & 43.88 & 0.80 & 0.06 & 1.00 & 0.08 \\
\hline 3 & 1.00 & 0.15 & 35 & 17.58 & 1.99 & 0.15 & 2.00 & 0.15 \\
\hline 4 & 1.00 & 0.15 & 35 & 6.76 & 5.18 & 0.40 & 6.00 & 0.46 \\
\hline 5 & 1.00 & 0.15 & 35 & 2.47 & 14.15 & 1.10 & 15.00 & 1.16 \\
\hline \multicolumn{9}{|c|}{ Marine propeller with 3 blades } \\
\hline 2 & 0.60 & 0.15 & 40 & 23.06 & 1.73 & 0.03 & 1.70 & 0.03 \\
\hline 3 & 0.60 & 0.15 & 40 & 8.73 & 4.58 & 0.08 & 4.00 & 0.07 \\
\hline 4 & 0.60 & 0.15 & 40 & 3.24 & 12.33 & 0.21 & 12.00 & 0.20 \\
\hline 5 & 0.60 & 0.15 & 40 & 1.16 & 34.45 & 0.58 & 30.00 & 0.50 \\
\hline \multicolumn{9}{|c|}{ Single turbine with 6 flat blades } \\
\hline 2 & 1.00 & 0.15 & 70 & 45.51 & 1.49 & 0.12 & 2.00 & 0.15 \\
\hline 3 & 1.00 & 15 & 70 & 18.51 & 3.78 & 0.29 & 4.00 & 0.31 \\
\hline 4 & 1.00 & 0.15 & 70 & 7.19 & 9.74 & 0.75 & 9.50 & 0.73 \\
\hline 5 & 1.00 & 0.15 & 70 & 2.47 & 28.31 & .19 & 0.00 & 80 \\
\hline
\end{tabular}

In order to analyse the dependence of power number Po on Reynolds number for shear-thinning CMC solutions it was plotted the graph in Figure 2.

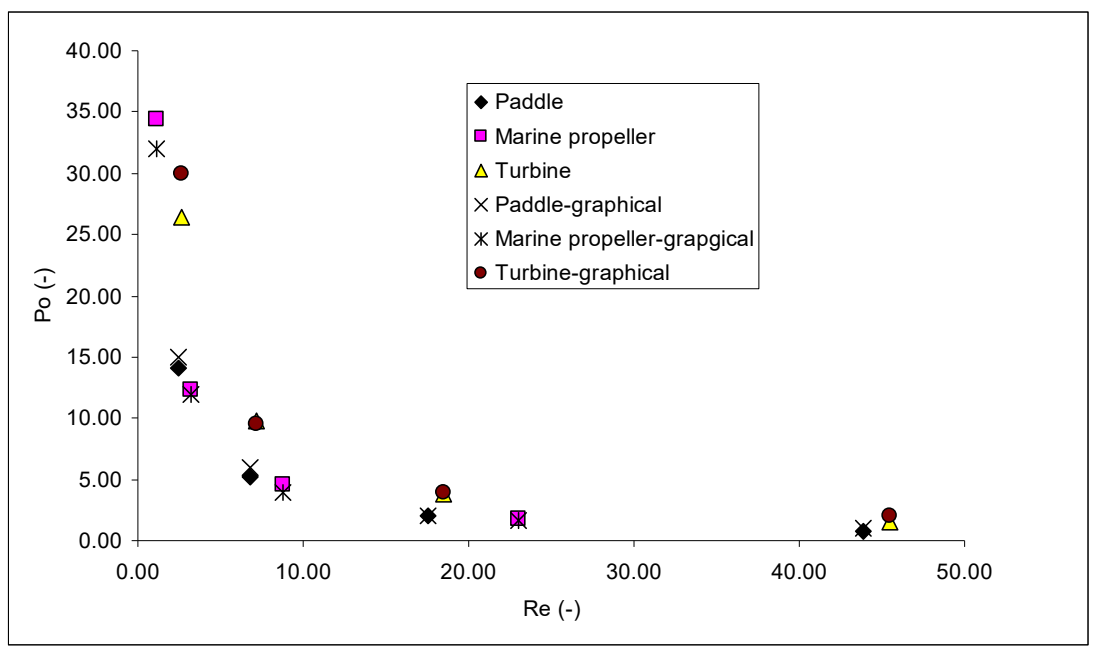

Figure 2. Dependence of Power number on Reynolds number 
The results from Figure 2 show the decrease of the power number Po with the increase of Reynolds number, as in the case of Newtonian fluids. Thus means an increase of power number and power consumption with the increase of material constancy $\mathrm{K}$, and decrease of flow index $\mathrm{n}$, in order to keep constant the impeller speed.

Although the values of power consumption are small, they are comparable with other values founded in literature [13, 14].

There are no high differences between values obtained by theoretical and graphical interpretation of the power number, the standard error $\varepsilon<10 \%$.

\section{CONCLUSIONS}

Based on the Metzner and Otto method, it was possible to estimate the mixing power requirements with reasonable accuracy in laminar regime, for CMC solutions, inelastic non-Newtonian fluids.

The value of Reynolds number has shown a decrease with the increase of material constancy $\mathrm{K}$ and with the decrease of flow index $\mathrm{n}$ for CMC solutions.

Power number and power consumption values were influenced by Reynolds number, especial by the apparent or effective viscosity.

The results have shown good agreement between theoretically calculated power number and the graphically determined values.

The obtained values of power consumption are small, but comparable with other values founded in literature.

\section{EXPERIMENTAL SECTION}

CMC solutions were prepared by dissolving carboxymethyl cellulose powder in an appropriated hot water and waiting sometime for it to swell, in order to obtain the desired concentration of each solution.

The rheological measurements were performed with a Rheotest-2 rotary rheometer with an S/S1 cylinder system, at an increasing and decreasing shear rate, ranging between $2.0-900 \mathrm{~s}-1$. The measurements were conducted at room temperature, $21 \pm 1^{\circ} \mathrm{C}$.

The flow curves were analyzed by power law or Ostwald de Vaele model in order to estimate the rheological parameters of CMC solutions, with a shear-thinning behaviour. 


\section{REFERENCES}

1. R.R. Chhabra; J.F. Richardson, Non-Newtonian Flow in the Process Industries. Fundamentals and Engineering Applications, 2nd ed.; Publisher: Butterworth Heinemann, Linacre House, Jordan Hill, Oxford, Great Britain, 2008; Chapter 8, pp. 325-358.

2. M. Cortada-Garcia; V. Dore; L. Mazzei; P. Angeli; Chem. Eng. Res. Des., 2017, 119, pp. 171-182.

3. G. Ascanio; B. Castro; E. Galindo; Chem. Eng. Res. Des., 2004, 82, 12821290.

4. A. Ghirisan; C.I. Anghel; S. Dragan; In Proceedings of the 41st International Conference of Slovak Society of Chemical Engineering, Editor: J. Markoš, Slovakia, 2014, pp. 863-872.

5. C. Chang; L. Zhang; Carbohyd. Polym., 2011, 84, 40-53.

6. C. Barba; D. Montane; X. Farriol; J. Desbrieres; M. Rinaudo; Cellulose, 2002, 9, 327-335.

7. A.B. Metzner; R.E. Otto; AIChEJ, 1957, 3, 3-10.

8. A.H.P. Skelland; in Handbook of Fluids in Motion, Edited by N. P. Cheremisinoff and R. Gupta, Ann Arbor Science, Ann Arbor, 1983, Chapter 7, pp. 623-860.

9. J.C. Godfrey; Static mixers, in Mixing in the Process Industries, Edited by N. Harnby, M.F. Edwards and A.W. Nienow, Butterworth-Heinemann, Oxford, $2^{\text {nd }}$ edition, 1992, Chapter 12, pp. 225-249.

10. J. Wang; L. Feng; X. GU; et al.; J. Chem. Eng. Sci., 2000, 55(12), 2339-2342.

11. D. Patel; F. Ein-Mozaffari; M. Mehrvar; Chem. Eng. Res. Des., 2015, 100, pp. 126-134.

12. Z.Y. Nor; A.A. H. Zafarizal; E.A.O. Nur; A.M. Yusrabbil, J. Oil Palm Research, 2020, in press Published online: 9 December 2020

13. D. Luan; Q. Chen; S. Zhou; Chin. J. Mech. Eng., 2014, 27(3), 635-640.

14. K. Yapici; B. Karasozen; M. Schaefer; Y. Uludag; Chem. Eng. Process: Process Intensification, 2008, 47, 1340-1349. 